\title{
Low temperature physics at room temperature in water: Charge inversion in chemical and biological systems
}

\author{
A.Yu. Grosberg, T.T. Nguyen, and B.I. Shklovskii \\ Department of Physics, University of Minnesota, 116 Church Street SE, Minneapolis, Minnesota 55455
}

\begin{abstract}
We review recent advances in the physics of strongly interacting charged systems functioning in water at room temperature. We concentrate on the phenomena which go beyond the framework of mean field theories, whether linear Debye-Hückel or non-linear Poisson-Boltzmann. We place major emphasis on charge inversion - a counterintuitive phenomenon in which a strongly charged particle, called macroion, binds so many counterions that its net charge changes sign. We discuss the universal theory of charge inversion based on the idea of a strongly correlated liquid of adsorbed counterions, similar to a Wigner crystal. This theory has a vast array of applications, particularly in biology and chemistry; for example, the DNA double helix in the presence of positive multivalent ions (e.g., polycations) acquires a net positive charge and drifts as a positive particle in electric field. This simplifies DNA uptake by the cell as needed for gene therapy, because the cell membrane is negatively charged. We discuss also the analogies of charge inversion in other fields of physics.
\end{abstract}

\section{CONTENTS}

I. Introduction

II. Historical remarks: Mean field theories

III. Strongly correlated liquid of multivalent ions

IV. Correlation-induced charge inversion

V. Enhancement of charge inversion by monovalent salt

VI. Screening of a charged plane by polyelectrolytes

VII. Polyelectrolytes wrapping around charged particles

VIII. Multilayer adsorption

IX. Correlation-induced attraction of like charges

X. Experimental evidence of charge inversion

XI. Correlations "in a sheep's skin"

XII. Charge inversion in a broader physics context

XIII. Conclusions and outlook

ACKNOWLEDGMENTS

References

\section{INTRODUCTION}

Molecular biological machinery functions in water at around room temperature. For a physicist, this very limited temperature range contrasts unfavorably to the richness of low temperature physics, where one can change temperature and scan vastly different energy scales in an orderly manner. In this Colloquium, we review the recently developed understanding of highly charged molecular systems in which Coulomb interactions are so strong that we are effectively in the realm of low temperature physics.

To be specific, imagine a problem in which one big ion, called a macroion, is screened by much smaller but still multivalent ions with a large charge $Z e$ each $(e$ is the proton charge); for brevity, we call them $Z$-ions. A variety of macroions are of importance in chemistry and biology, ranging from the charged surface of mica or charged solid particles, to charged lipid membranes, colloids, DNA, actin, and even to cells and viruses. Multivalent metal ions, charged micelles, dendrimers, short or long polyelectrolytes including DNA - to name but a few - can play the role of the screening $Z$-ions.

The central idea of this Colloquium is that of correlations: due to strong interactions with the macroion surface and with each other, screening $Z$-ions do not position themselves randomly in three-dimensional space, but form a strongly correlated liquid on the surface of the macroion. Moreover, in terms of short range order this liquid is reminiscent of a Wigner crystal, as the cartoon in Fig. 1 depicts. Because of its central importance we shall use special abbreviation SCL to denote a strongly correlated liquid of adsorbed $Z$-ions.

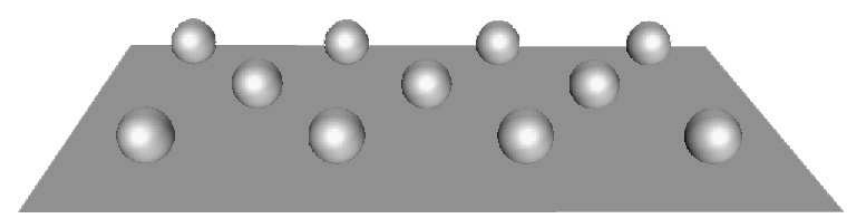

FIG. 1. Strongly correlated liquid (SCL) - almost a Wigner crystal - of $Z$-ions on the oppositely charged macroion surface. The figure is characteristic in showing the degree to which we are willing to ignore the microscopic details.

Depending on the system geometry and other circumstances, correlations between screening ions may appear in many different ways. To create some simple images in the reader's mind, it is useful to begin with a few examples. One example is that in Fig. 1, which may be the surface of, e.g., a latex particle screened by some compact ions. With a modest leap of the imagination, we can envision the same picture to represent the surface of a DNA double helix screened by multivalent counterions, such as spermine with $Z=4$ (Bloomfield, 1996). Here, we imagine DNA as a thick and long cylinder, diameter 
$2 \mathrm{~nm}$ and charge $-e$ per $1.7 \mathrm{~nm}$ along the cylinder, or, in other words, with a huge negative surface charge density $-0.9 \mathrm{e} / \mathrm{nm}^{2}$. We study correlations between point-like $Z$-ions in Secs. III - V. One obvious problem with the model Fig. 1 is that it ignores the discreteness of charges, both in the macroion and in the $Z$-ions; chemists, for instance, hardly ever accept such continuous models. In Sec. XI, we address the electrostatic correlations in the systems with discrete charges.

As another example, consider DNA molecules which screen the positive surface charge of a colloid particle. Obviously, DNA chains play the role of $Z$-ions. For very short DNA pieces we are back again to Fig. 1, but longer DNA are spaghetti-like, and in this situation, correlations mean parallel arrangement, as we see in Fig. 2. Theoretical treatment of this problem in Sec. VI makes a simplifying assumption that it may be modeled as a system of parallel rods.

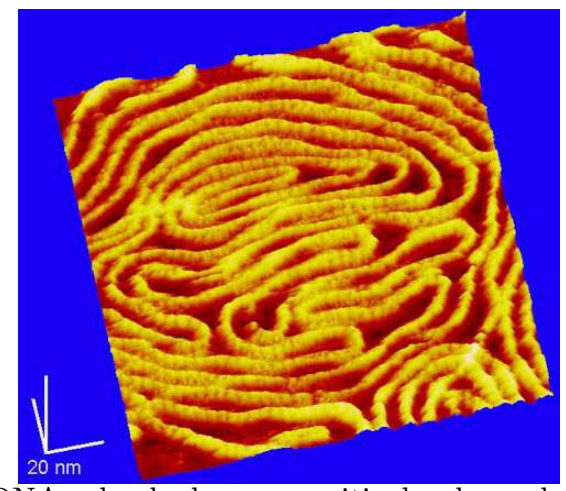

FIG. 2. DNA adsorbed on a positively charged surface, as seen in an atomic force microscopy image (Mou et al, 1995).

Electrostatic correlations are strong not only for artificial systems involving DNA, but also for the DNA in the cell (Aberts et al, 1994). In particular, to organize DNA in chromatin (in eukariotic cells), nature uses proteins having large positive charges - histones. Higher levels of chromatin hierarchical structure can be disassembled, with some of the histones released, by an elevated concentration of salt. The resulting most stable lower level structure is a bead-on-a-string necklace, as shown in the Fig. 3. It is called $10 \mathrm{~nm}$ fiber, because the beads, called nucleosomes, are about that big. Each nucleosome consists of a core particle, called an octamer, with a total charge of about $+170 e$. As shown in the inset in Fig. 3 , the octamer is encircled 1.8 times by a DNA having a charge $-292 e$. The electrostatic interaction energy between the DNA molecule and the histone octamer dwarfs the bending energy of the DNA molecule. Strikingly, a simple theory discussed in Sec. VII yields a similar structure, Fig. 4, based on purely electrostatic correlations in a simple model.

Correlated screening has also many useful practical applications. The first to be mentioned is the technology of dressing of DNA with polycations (Kabanov and Kabanov, 1995 and 1998), positively charged star-like polymers called dendrimers (Tang et al, 1996; Kabanov et al, 2000; Evans et al, 2001), or liposomes (Radler et al, 1997) in order to produce positive complexes with DNA. This facilitates gene delivery through a negative cell surface membrane (Felgner, 1997). There is also the idea to manufacture nano-wires by attaching positive silver or gold colloids to DNA (Braun et al, 1998; Keren et al, 2001).

We view these examples as both important and convincing enough to engage in the study of electrostatic correlations between strongly charged $Z$-ions. Strong correlations manifest themselves in a number of ways and alter dramatically the whole picture of screening. In the familiar Debye theory, screening somewhat reduces the effective value of charge as seen from a finite distance in the outside world. With strongly correlated ions, overscreening becomes possible, in which case the shielded macroion charge is seen from outside as having the opposite sign. This counterintuitive phenomenon is called charge inversion.

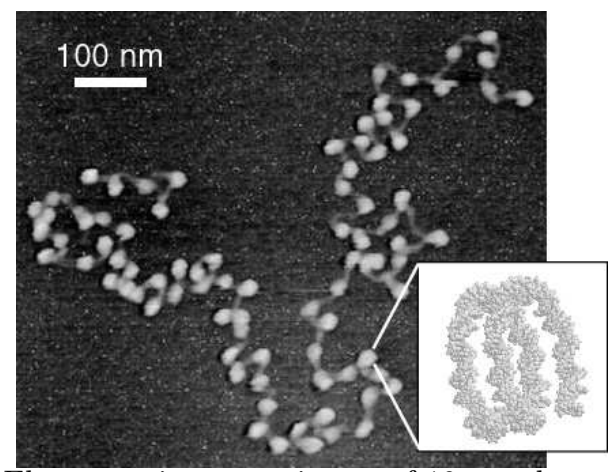

FIG. 3. Electron microscopy image of $10 \mathrm{~nm}$ chromatin fiber (Shao, 1999). The beads are nucleosomes. The structure of a nucleosome is known to about $0.2 \mathrm{~nm}$ resolution (Luger et al, 1997), and the inset shows how the DNA double helix is bent in the nucleosome. While the overall shape of the fiber is perhaps due to the sample preparation procedure, the array of nucleosomes on the DNA is close to periodic, similar in this respect to the theoretical model shown in Fig. 4.

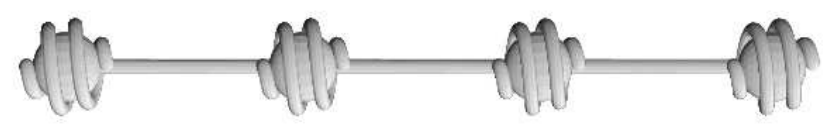

FIG. 4. Self-assembled complex of a negative polyelectrolyte molecule and many positive spheres in a necklace-like structure. On the surface of a sphere, neighboring polyelectrolyte turns are correlated similar to the rods in Fig. 8 below. On a larger scale, charged spheres repel each other and form one-dimensional Wigner crystal along the polyelectrolyte molecule. 
The first experimental study related to charge inversion was reported a long time ago by De Jong (1949). More recently, charge inverted complexes of polyelectrolytes were directly observed in electrophoresis experiments (see review articles by Kabanov and Kabanov (1995 and 1998) and references therein). It is now understood that charge inversion is a generic phenomenon and it is expected in all systems in which strongly charged ions participate in screening. It turns out to be a natural manifestation of correlations between screening ions. In recent years, the phenomenon of charge inversion has attracted significant attention of theorists (Ennis et al, 1996; Wallin and Linse, 1996; Perel and Shklovskii, 1999; Shklovskii, 1999b; Mateescu et al, 1999; Park et al, 1999; Joanny, 1999; Sens and Gurovich, 1999; Netz and Joanny, 1999a; Netz and Joanny, 1999b; Wang et al, 1999; Nguyen et al, 2000a-b; Messina et al, 2000; Nguyen and Shklovskii, 2001a-e; Chodanowski and Stoll, 2001; Dobrynin et al, 2001; Andelman and Joanny, 2000; Potemkin et al, 2001; Tanaka and Grosberg, 2001a.)

Another equally interesting manifestation of correlations is the possibility of attraction between like charged macroions mediated by $Z$-ions (Rouzina and Bloomfield, 1996; Gronbech-Jensen et al, 1997; Levin et al, 1999; Moreira and Netz, 2000b). This has implications in the large field of self-assembly of charged biological objects, ranging from RNA (Woodson, 2000; Pan et al, 1999) to virus heads (Gelbart et al, 2000). In Sec. IX, we show how this attraction of like charges competes with repulsion due to inverted charges, which combine to induce reentrant condensation of DNA or colloids out of solutions.

Although the subject matter of our Colloquium belongs to chemical and biological physics, it has remarkably many deep physical analogies in other branches of physics, from atomic physics to quantum Hall effect; we discuss this in Sec. XII.

In what follows, we re-examine the entire concept of screening to include correlations. Although this requires stepping beyond the mean field approximation, we shall start with some historical remarks which also serve to define the notations.

\section{HISTORICAL REMARKS: MEAN FIELD THEORIES}

The history of our subject is almost one century long. While we are unable in this brief Colloquium to discuss it in any depth, we shall mention three key steps.

In the first step, Gouy (1910) and independently Chapman (1913) examined the double layer at an electrode surface, an intensely disputed subject at the time. Following Gouy and Chapman, let us consider a massive insulating macroion. To be specific, assume that its charge is negative, with surface density $-\sigma$. Assume further that the only counterions in the system are those dissociated from the surface. Since a macroion is large, the problem of the counterion distribution near the surface is one-dimensional, with both counterion concentration $N(x)$ and electrostatic potential $\phi(x)$ depending on the distance $x$ from the surface. Gouy and Chapman addressed this problem by solving the following equation:

$$
\Delta \phi=-\frac{4 \pi}{\epsilon} N_{s} e Z \exp \left[-e Z \frac{\phi(x)-\phi_{s}}{k_{B} T}\right] .
$$

Here $\epsilon \approx 80$ is the dielectric constant of water, $\phi_{s}$ and $N_{s}$ are the potential and counterion concentration at the surface. Formula (1) follows from the Poisson equation for the potential $\phi(x)$ and the assumption that the ions determining the charge density are Boltzmann-distributed in the same potential. Because of this self-consistency assumption, this equation describes a mean field approximation. It is universally called the Poisson-Boltzmann equation. One boundary condition $d \phi /\left.d x\right|_{x=0}=4 \pi \sigma / \epsilon$ follows from the fact that the field vanishes on the other side of the boundary (as there is no electrolyte and there are no charges). The second condition is that the concentration $N(x)$ must be normalized to $\sigma / Z e$ ions per unit area. The exact solution of the thus formulated GouyChapman problem reads

$$
N(x)=\frac{k_{B} T \epsilon / 2 \pi e^{2} Z^{2}}{(x+\lambda)^{2}},
$$

where $\lambda$ is called Gouy-Chapman length, which is equal to

$$
\lambda=k_{B} T \epsilon / 2 \pi \sigma Z e .
$$

The interpretation of Eq. (3) is interesting. If a $Z$-ion were to be alone next to the charged plane, it would be confined by the surface field $2 \pi \sigma / \epsilon$ to such a "height" $\lambda$ that its energy change $2 \pi \sigma Z e \lambda / \epsilon$ is about $k_{B} T$ - which leads to the correct answer Eq. (3). Other $Z$-ions cancel the field inside the macroion but double the field in the electrolyte at the macroion surface (see above the boundary condition at $x=0$ ). On the other hand, every particular ion at every moment is higher than, roughly, half the other ions, whereupon it finds itself in a partially screened field. Exact solution of the Poisson-Boltzmann equation indicates that these two factors cancel each other, yielding Eq. (3).

The next step of the screening story, well known to every physicist, is the theory of Debye and Hückel (1923) initially developed for electrolytes - the overall neutral mixture of mobile ions of both signs, and now widely used in plasma and solid state physics. Debye and Hückel linearized the Poisson-Boltzmann equation (1) (generalized by introducing the sum over ion species on the right-hand side). Of course, linearization can be done if the potential is not too strong anywhere in the system, which is often 
the case if charges involved are small enough. DebyeHückel screening leads to exponential decay of the potential around a point-like charge $Q$ :

$$
\phi(r)=\frac{Q}{\epsilon r} e^{-r / r_{s}},
$$

where the Debye-Hückel radius is given by

$$
r_{s}=\left(\frac{k_{B} T}{8 \pi N_{1} e^{2}}\right)^{1 / 2}
$$

and $N_{1}$ is the concentration of monovalent salt.

Less well known among physicists is the fact that Debye-Hückel theory ignited a heated debate: Bjerrum (1926) commented that $\int \exp \left[e^{2} / r k_{B} T\right] r^{2} d r$ diverges at $r \rightarrow 0$ and, therefore, point-like charged particles are expected to associate in neutral pairs. The discussion led to the realization of the important role of short range repelling forces. In order to prevent association of monovalent ions and formation of Bjerrum pairs, the repulsion forces should take over at the distance which is not much smaller than the so-called Bjerrum length

$$
\ell_{B}=e^{2} / \epsilon k_{B} T,
$$

which is about $0.7 \mathrm{~nm}$ in water at room temperature. (This is why hydration layer of a few water molecules around each ion is essential to stabilize dissociated ions).

The last step we mention here is relatively recent, it has to do with non-linear screening of cylindrical charges, such as the DNA double helix (Onsager, 1967; Manning, 1969; Oosawa, 1971). Consider a cylinder charged to the linear density $-\eta$. Since the potential is logarithmic, its competition with entropy is quite peculiar. Indeed, releasing counterions to some distance $r$ requires energy $(2 e Z \eta / \epsilon) \ln (r / a)$ ( $a$ being the cylinder radius), while the corresponding entropy gain is $k_{B} T \ln \left(\pi r^{2} / \pi a^{2}\right)$. Therefore, counterions are released only as long as $\eta<\eta_{Z}$, where

$$
\eta_{Z}=k_{B} T \epsilon / e Z .
$$

When a cylinder is charged in excess of $-\eta_{Z}$, some of the ions remain Onsager-Manning condensed on the cylinder, so that its effective net charge is equal to $-\eta_{Z}$. To emphasize the importance of this subject, let us mention that the DNA double helix has a bare charge density of about $-4.2 \eta_{1}$, where $\eta_{1}=\left.\eta_{Z}\right|_{Z=1}$ (see FrankKamenetskii et al (1987) for further DNA applications). Onsager-Manning condensation was more accurately justified by Zimm and Le Bret (1983). These authors addressed non-linear Poisson-Boltzmann equation in cylindrical geometry (refining earlier works - see the collection of papers Katzir-Katchalsky (1971)).

Gouy-Chapman, Debye-Hückel, and Onsager-Manning theories are all of mean field type, based on the PoissonBoltzmann equation. This approach works well when screening charges are small, $Z=1$. In the case of strongly charged macroions and $Z$-ions with $Z \gg 1$, however, correlations are important and mean field theory fails. Hence it is necessary to step beyond this approximation. This is precisely the subject matter of the present Colloquium. Charge inversion in this context should be viewed as the most obvious manifestation of the failure of the mean field approximation.

\section{STRONGLY CORRELATED LIQUID OF MULTIVALENT IONS}

To begin with, let us explain why the Gouy-Chapman solution (2) fails at large $Z$. Apart from $\lambda$ (3), there is a second length scale in the problem due to the discreteness of charge. It is associated with the distance between ions in the lateral direction, along the plane. As long as the system as a whole is neutral, the two-dimensional concentration of $Z$-ions is $n=\sigma / Z e$, and the surface area per ion can be characterized by a radius $R$ such that $\pi R^{2}=1 / n$ (see Fig. 5). Thus $R=(\pi n)^{-1 / 2}=$ $(Z e / \pi \sigma)^{1 / 2}$, and hence (cf. Eq. (3)

$$
\frac{R}{\lambda}=2 \Gamma, \quad \Gamma=\frac{Z^{2} e^{2} / \epsilon R}{k_{B} T} .
$$

Here $\Gamma$ is the Coulomb coupling constant, or the inverse dimensionless temperature measured in the units of a typical interaction energy between $Z$-ions. A system of monovalent ions, $Z=1$, is weakly coupled, $\Gamma \sim 1$, this is why classical mean field theory applies. By contrast, a system in which $Z$-ions have large $Z$ is strongly coupled, and we see that $R$ becomes larger than $\lambda$. For example, at $Z=3$ and $\sigma=1.0 \mathrm{e} / \mathrm{nm}^{2}$ we get $\Gamma=6.4$, $\lambda \simeq 0.1 \mathrm{~nm}$ and $R \simeq 1.0 \mathrm{~nm}$. Clearly, mean field treatment along the lines of Poisson-Boltzmann theory fails in this situation, since $Z$-ions do not affect each other when they are at distances smaller than $R$ from the plane. It is worth emphasizing once again that it is not only the linearized Debye-Hückel theory that fails in the strong coupling regime, but so does also the non-linear PoissonBoltzmann theory. It is the mean field approximation that is inapplicable because of correlations between discreet charges.

An alternative theory appropriate for the regime $\Gamma \gg$ 1 was suggested by Perel and Shklovskii (1999). The main idea of this theory is that at $\Gamma \gg 1$ the screening atmosphere is narrowly confined at the surface (see Fig. 1 ), and it should be approximated as a two-dimensional SCL.

A two-dimensional liquid of classical charged particles on a neutralizing background, the so-called one component plasma, is well understood (Totsuji, 1978). At zero temperature, it acquires the minimal energy state of a 
Wigner crystal, shown in the Fig. 5, in which the correlation energy per ion and the chemical potential are given by

$$
\begin{aligned}
& \varepsilon(n) \simeq-1.11 Z^{2} e^{2} / R \epsilon=-1.96 n^{1 / 2} Z^{2} e^{2} / \epsilon, \\
& \mu_{W C}=\frac{\partial[n \varepsilon(n)]}{\partial n}=\frac{3}{2} \varepsilon(n)=-1.65 Z^{2} e^{2} / \epsilon R .
\end{aligned}
$$

We interpret $R$ here as the radius of a Wigner-Seitz cell approximated by a disc (see Fig. 5).

At non-zero temperature, the chemical potential of one component plasma can be written as $\mu=\mu_{i d}+\mu_{W C}+$ $\delta \mu$. Here $\mu_{i d}$ is the chemical potential of an ideal gas at the same concentration. Accordingly, $\mu_{W C}+\delta \mu$ part is entirely due to correlations. Furthermore, it turns out that $\delta \mu$, which is the thermal correction, is negligible for $\Gamma \gg 1$. Although a Wigner crystal, in terms of long range order, melts at $\Gamma \approx 130$, the value of $\delta \mu$ is controlled by short range order and remains negligible as long as $\Gamma \gg 1$. It is in this sense that a SCL of $Z$-ions is similar to a Wigner crystal.

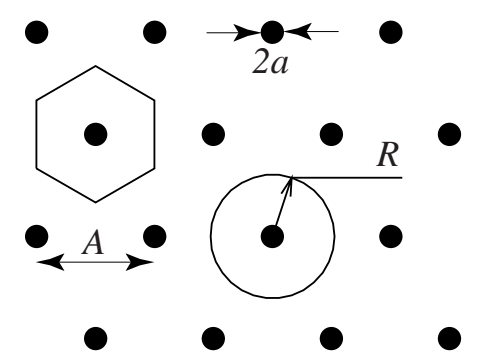

FIG. 5. A Wigner crystal of positive $Z$-ions on a uniform background of negative surface charge. A hexagonal WignerSeitz cell and its simplified version as a disk with radius $R$ are shown.

Thus, the correlation part of the chemical potential can be approximated by $\mu_{W C}(10)$, which is negative and large: $-\mu_{W C} / k_{B} T=1.65 \Gamma \gg 1$. The physics of a large and negative $\mu_{W C}$ can be understood as follows. Pretend for a moment that the insulating macroion is replaced by a neutral metallic particle. In this case, each $Z$-ion creates an image charge of opposite sign inside the metal. The energy of attraction to the image is $U(x)=-(Z e)^{2} / 4 \epsilon x$, where $x$ is the distance to the surface. This energy is minimal when the $Z$-ion is placed next to the surface, at a distance equal to its radius $a$; therefore, the $Z$-ion sticks to the surface. With this idea in mind, consider bringing a new $Z$-ion to the insulating macroion surface already covered by an adsorbed layer of $Z$-ions (Fig. 6). This layer behaves like a metal surface in the sense that the new $Z$-ion repels adsorbed ones, creating a correlation hole. In other words, it creates a negative image. Because of the discreteness of charges, the adsorbed layer is a good metal at length scales above $R$ only. Accordingly, attraction to the image gets saturated at $x \sim R$. This is why the chemical potential of an ion in a Wigner crystal scales as $\mu_{W C} \sim-(Z e)^{2} / \epsilon R$. Eq. (10) specifies the numerical coefficient in this expression.

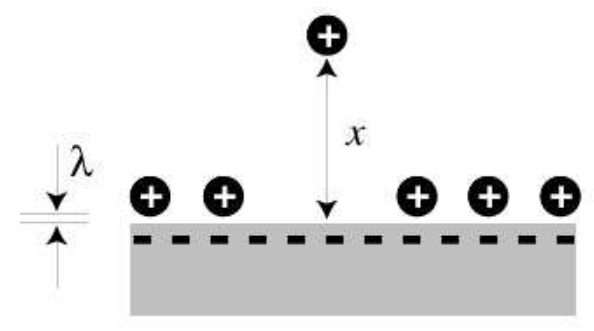

FIG. 6. The origin of attraction of a new positive $Z$-ion to the already neutralized surface. $Z$-ions are shown by solid circles. The new $Z$-ion creates its negative correlation hole.

Using the concept of images, we can now understand the distribution of $Z$-ions, $N(x)$, near the surface. To do this, let us extract one $Z$-ion from the SCL and move it along the $x$ axis. As long as $x \ll R$, its correlation hole does not change, and, therefore, the $Z$-ion is attracted to the surface by the uniform electric field $E=2 \pi \sigma / \epsilon$; other $Z$-ions do not affect this attraction in any way. Therefore, $N(x)=N_{s} \exp (-x / \lambda)$ for $x \ll R$. Here $N_{s} \simeq n / \lambda$ is the three-dimensional concentration of $Z$ ions close to the surface plane (Eq. (1)). For $x \gg R$, the correlation hole acts as a point-like image charge, the corresponding interaction energy being $-Z^{2} e^{2} / 4 \epsilon x$. At $x=Z^{2} e^{2} / 4 \epsilon k_{B} T=R_{0} \Gamma / 4=\lambda \Gamma^{2} / 2$, the interaction with the image charge drops to about $k_{B} T$, i.e., negligible, and the $Z$-ion concentration becomes

$$
N_{0}=N_{s} \exp \left(-\frac{\left|\mu_{W C}\right|}{k_{B} T}\right)=N_{s} \exp \left(-\frac{1.65 Z^{2} e^{2}}{\epsilon R k_{B} T}\right)
$$

We shall further comment on the physical meaning of $N_{0}$ after Eq. (12). Note that the correction term $-Z^{2} e^{2} / 4 \epsilon x$ to $Z$-ion energy, which is important in the interval $R \ll$ $x \ll \lambda \Gamma^{2} / 2$, is similar to the "image" correction to the work function of a metal (Lang, 1973).

The dramatic difference between the exponential decay of $N(x)$ and the Gouy-Chapman $1 /(\lambda+x)^{2}$-law (2) is due to correlation effects. Moreira and Netz (2000a) rederived these results in a more formal way and confirmed them by Monte-Carlo simulations. Recently, Moreira and Netz (2001) also showed that discreteness of the surface charge neglected above leads to the lateral pinning of $Z$ ions. This brings $Z$-ions even somewhat closer to the surface. General direction of this effect can be understood from the limit (although unrealistic for strongly charged surface) when the distance of closest approach between a discrete surface charge and a $Z$-ion is so small that they form isolated Bjerrum pairs (see (6)).

At larger $x$, correlations and interactions with image charges are unimportant, and the Poisson-Boltzmann 
equation applies. In this region $N(x)$ varies so smoothly that $N(x)=N_{0}$ provides an effective boundary condition for the Poisson-Boltzmann equation (Shklovskii, 1999b). At this stage we must remember that in a real physical situation there is always some concentration of $Z$-ions, $N$, in the surrounding solution. It can be either larger or smaller than $N_{0}$. In the latter case, the surface is overcharged, as we show in Sec. IV below.

\section{CORRELATION-INDUCED CHARGE INVERSION}

Let us return once again to the physical argument illustrated by Fig. 6. It explains why an extra $Z$-ion may be attracted to the macroion surface despite the fact that it is already neutralized by the previously adsorbed $Z$-ions. What happens if another $Z$-ion approaches? Clearly, correlation effect will keep providing an attractive force for this and subsequent $Z$-ions, but it will have to compete with the repulsive force which is simply due to the fact that macroion has already too many $Z$-ions adsorbed and the whole complex is, therefore, positively charged. Thus, the question is this: what is the equilibrium amount of (over)charge?

One useful way to think about it is to realize that correlation mechanism provides voltage to drive overcharging, but the actual amount of (over)charge depends on both voltage and capacitance. Since the latter depends strongly on the geometry, we will have to explore several cases - spherical macroion, cylindrical, etc.

Another, equivalent, view, involves the comparison of chemical potentials of adsorbed $Z$-ions and $Z$-ions in the bulk solution. This approach immediately suggests that in equilibrium the total charge, $Q^{*}$, depends on the concentration, $N$, of $Z$-ions in the surrounding bulk solution. Here $Q^{*}$ is the net charge of the entire complex which includes bare charge of the macroion, $-Q<0$, and the proper (determined by the equilibrium condition) number of adsorbed $Z$-ions with charge $Z e>0$ each.

Let us see now how we can implement the condition of equal chemical potentials for the case of a spherical macroion, with radius $r$. As regards adsorbed $Z$-ions, we $\operatorname{argue}^{1}$ that their chemical potential is only different from

\footnotetext{
${ }^{1}$ The argument goes as follows. Spherical surface of the macroion has bare surface charge density $-\sigma=-Q / 4 \pi r^{2}$. Let us pretend to place there, along with real charge $-\sigma$, also two imaginary spheres, with uniform charge densities $\sigma^{*}=Q^{*} / 4 \pi r^{2}$ and $-\sigma^{*}$. Their total charge is zero, so they have no effect. However, we can now think that the $Z$-ions are adsorbed on the sphere with the charge density $-\sigma-\sigma^{*}$, and with this sphere they form a neutral SCL, quite like that considered in Sec. III. The remaining sphere has the charge density $\sigma^{*}$ and creates spherically symmetric field with potential
}

the one-component plasma expression (10) by the energy of the $Z$-ion in the potential $\psi(0)=Q^{*} / \epsilon r$ created by the net charge $Q^{*}$. Therefore, equilibrium condition reads $\mu_{i d}+\mu_{W C}+Z e \psi(0)=\mu_{b}$, where $\mu_{b}$ is the bulk chemical potential. To determine $Q^{*}$ from here, we first note that $\mu_{i d}-\mu_{b}=k_{B} T \ln \left(N_{s} / N\right)$; we further express $\mu_{W C}$ in terms of $N_{0}$ (see Eq. (11)), and finally obtain

$$
Q^{*}=\frac{\epsilon r}{Z e} k_{B} T \ln \left(N / N_{0}\right) .
$$

Clearly, the net charge $Q^{*}$ is indeed positive when $N>$ $N_{0}$, i.e. it has the sign opposite to the bare charge $Q$.

The result (12) sheds also light on the meaning of the quantity $N_{0}$ defined above, in Eq. (11): this is the concentration of $Z$-ions in the surrounding bulk solution at which macroion is exactly neutralized by the adsorbed $Z$-ions. This concentration is very small because $\left|\mu_{W C}\right| / k_{B} T \gg 1$. For example, $N_{0}=0.3 \mathrm{mM}$ and $0.8 \mu \mathrm{M}$ for $Z=3$ and 4 respectively $\left(1 \mathrm{M} \approx 6 \times 10^{20} \mathrm{~cm}^{-3}\right)$. Therefore, it is easy to increase charge inversion by increasing $N$. How far does it go? At large enough $N$, translational entropy terms $\mu_{b}-\mu_{i d}$ become negligible compared to $\mu_{W C}$, yielding

$$
Q^{*} / \epsilon r=\psi(0)=\left|\mu_{W C}\right| / Z e .
$$

Expressing $R$ and $\left|\mu_{W C}\right|$ through $Q$ and $Z$ with the help of Eq. (10) (and remembering that $\sigma^{*}=-\sigma+Z e n$ ), Shklovskii (1999b) arrived at the prediction for the $\max$ imal inverted charge for the spherical macroion which can be achieved by increasing concentration of $Z: 1$ salt:

$$
Q^{*}=0.83 \sqrt{Q Z e} .
$$

This charge is much larger than $Z e$, but still is smaller than $Q$ because of limitations imposed by the large charging energy. For example, for $Q=100 e, Z=4$, we get $Q^{*}=17 e$. Eq. (14) was recently confirmed by numerical simulations (Messina et al, 2000; Tanaka and Grosberg, 2001a). Further increase of charge inversion beyond the level dictated by Eq. (14) is achievable with the help of a monovalent salt (see Sec V).

Applied in cylindrical geometry, similar arguments lead to a revision of the conventional Onsager-Manning condensation theory (Sec. II) when dealing with multivalent $Z$-ions. Consider a cylinder with a negative linear charge density $-\eta$ and assume that $\eta>\eta_{Z}$. Mean field Onsager-Manning theory (7) predicts $\eta^{*}=-\eta_{Z}$. By contrast, Perel and Shklovskii (1999) showed that a

on the surface $\psi(0)$. We emphasize that the macroscopic net charge $\sigma^{*}$ does not interact with the one component plasma, because the potential $\psi(0)$ is constant along the surface, while one component plasma is neutral. 
correlation-induced negative chemical potential $\mu_{W C}$ results in

$$
\eta^{*}=-\eta_{Z} \frac{\ln \left(N_{0} / N\right)}{\ln \left(4 / \pi Z^{6} N \ell_{B}^{3}\right)},
$$

where $\ell_{B}$ is the Bjerrum length (6). This result reproduces the Onsager-Manning one (7) only at extremely small values of $N$, which are unrealistic at $Z \geq 3$. On the other hand, at $N=N_{0}$ the net charge flips sign, resulting in charge inversion at $N>N_{0}$ (which is absent in Onsager-Manning theory). At large enough $N$, the inverted charge density $\eta^{*}$ can reach $k_{B} T \epsilon / e=\eta_{1}$.

\section{ENHANCEMENT OF CHARGE INVERSION BY MONOVALENT SALT}

Most water solutions, particularly biological ones, contain significant amounts of monovalent salt, such as $\mathrm{NaCl}$. Correlations between these monovalent ions are negligible, and, therefore, their only role is to provide Debye-Hückel screening with a decay length $r_{s}(5)$. This screening makes charge inversion substantially stronger. Indeed, screening by a monovalent salt diminishes the charging energy of the macroion much stronger than the correlation energy of $Z$-ions. Furthermore, in a sufficient concentration of salt, the macroion is screened at the distance smaller than its size. Then, the macroion can be thought of as an over-screened surface, with inverted charge $Q^{*}$ proportional to the surface area. In this sense, overall shape of the macroion is irrelevant, at least to a first approximation. Therefore, we consider here a simpler case: screening of a planar macroion surface with a negative surface charge density $-\sigma$ by a solution with a concentration $N$ of $Z: 1$ salt and a large concentration $N_{1}$ of a monovalent salt.

Nguyen et al (2000a) calculated analytically the dependence of the charge inversion ratio, $\sigma^{*} / \sigma$, on $r_{s}$, in two limiting cases $r_{s} \gg R_{0}$ and $r_{s} \ll R_{0}$, where $R_{0}=(\pi \sigma / Z e)^{-1 / 2}$ is the radius of a Wigner-Seitz cell at the neutral point $n=\sigma / Z e$. At $r_{s} \gg R_{0}$ calculation starts from Eq. (13). The electrostatic potential of a plane with the charge density $\sigma^{*}$ screened at the distance $r_{s}$ reads $\psi(0)=4 \pi \sigma^{*} r_{s}$. At $r_{s} \gg R_{0}$ screening by monovalent ions does not change Eq. (10) substantially so that we still can use it in Eq. (13) which now describes charging of a plane capacitor by voltage $\left|\mu_{W C}\right| / Z e$. This gives

$$
\sigma^{*} / \sigma=0.41\left(R_{0} / r_{s}\right) \ll 1 \quad\left(r_{s} \gg R_{0}\right) .
$$

Thus, at $r_{s} \gg R_{0}$ inverted charge density grows with decreasing $r_{s}$.

Now we switch to the case of strong screening by monovalent salt. To begin with, let us assume that screening is already so strong that $r_{s} \ll R_{0}$, but energy of SCL is still much greater than $k_{B} T$ per $Z$-ion. In this regime, free energy consists of Debye-Hückel screened nearest neighbor repulsion energies of $Z$-ions and the attraction energy of $Z$-ions to the charged surface:

$$
F=\left(3 n Z^{2} e^{2} / \epsilon A\right) \exp \left(-A / r_{s}\right)-4 \pi \sigma r_{s} Z e n / \epsilon,
$$

where $A=(2 / \sqrt{3})^{1 / 2} n^{-1 / 2}$ is the lattice constant of the hexagonal Wigner crystal (Fig. 5). Minimizing $F$ with respect to $n$ one arrives at

$$
\frac{\sigma^{*}}{\sigma}=\frac{\pi}{2 \sqrt{3}}\left(\frac{R_{0}}{r_{s} \ln \left(R_{0} / r_{s}\right)}\right)^{2} \quad\left(r_{s} \ll R_{0}\right) .
$$

Thus $\sigma^{*} / \sigma$ grows with decreasing $r_{s}$ and can become larger than $100 \%$. At $r_{s} \sim R_{0}$, Eq. (16) and Eq. (18) match each other. As we see, $\sigma^{*}$ continues to grow with decreasing $r_{s}$. This is because the repulsion between $Z$ ions becomes weaker, so that it is easier to pack more $Z$-ions on the surface. Of course, when $r_{s}$ decreases even further, binding energy of $Z$-ions becomes small, SCL dissolves, and charge inversion disappears.

The results above are in a good agreement with simulations. Terao and Nakayama (2001) reported the results of a Monte Carlo simulation for the system consisting of a macroion with charge $Q=20 e$ surrounded by 20 monovalent counterions and 1500 ions of $2: 1$ electrolyte. Tanaka and Grosberg (2001a) performed molecular dynamics simulations with a spherical macroion of the charge $Q=-28 e$, spherical $Z$-ions $(2 \leq Z \leq 7)$, and up to 500 monovalent ions; the system is neutral overall, which determines the number of $Z$-ions to be between 180 and 52. Simulations confirmed the strong adsorption of the overcharging amount of $Z$-ions on the surface of macroion. On a more quantitative level, Tanaka and Grosberg (2001a) examined the dependence of the inverted charge on the ionic strength, $I$, and found the crossover between $Q^{*} \propto \sqrt{I} \propto 1 / r_{s}$ and $Q^{*} \propto I \propto 1 / r_{s}^{2}$, consistent with Eqs. (16) and (18). Tanaka and Grosberg (2001a) attempted also to maximize the charge inversion ratio $Q^{*} / Q$. In agreement with the theoretical views presented above, the growth of charge inversion is capped when correlations between $Z$-ions are suppressed. We have mentioned eventual reduction of correlations for large monovalent salt concentration, or small $r_{s}$, when the SCL evaporates. If one tries to increase $Z$ instead of lowering $r_{s}$, then, at large $Z$, correlations become suppressed because monovalent ions condense on $Z$-ions, forming $Z^{\prime}$ ions with smaller net charge $Z^{\prime}$. This effect is clearly seen in Fig.7 (see Nguyen et al (2000b) for the conditions under which this phenomenon is and is not important). Nevertheless, $Q^{*} / Q$ up to about $150 \%$ is easily observed. 


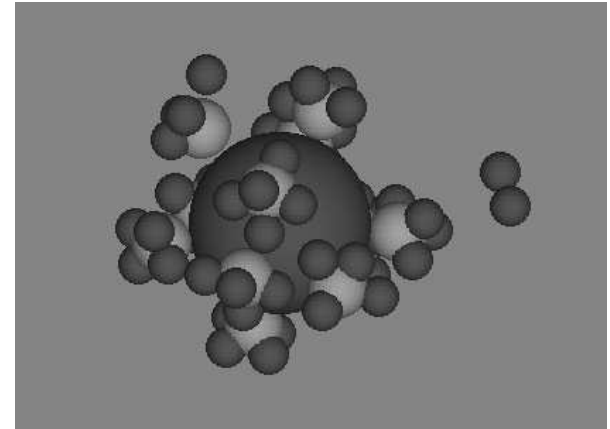

FIG. 7. The snapshot of the system simulated by Tanaka and Grosberg (2001a). The spherical macroion is grey, $Z$ ions are white, and monovalent negative ions are black. Bare charge of the spherical macroion is $Q=-28 e, Z=7$, and $\Gamma \approx 30$. There are $52 Z$-ions and 336 monovalent ions in the simulation domain. What is clearly seen is the formation of $Z$-ions: because $Z$ is so large, $Z$-ions adsorb monovalent ions. This reduces correlations between $Z$-ions and restricts charge inversion. Nevertheless, bare charge of the complex shown in the figure is $+16 e$.

\section{SCREENING OF A CHARGED PLANE BY POLYELECTROLYTES}

A practically important class of $Z$-ions are charged polymers, i.e., polyelectrolytes. Let us start with a rigid polyelectrolyte and discuss charge inversion caused by adsorption of long rod-like $Z$-ions. For example, the moderately long (up to about $50 \mathrm{~nm}$, or about 150 base pairs) DNA double helix can be well approximated as a rod. Actin is another example of an even more rigid polyelectrolyte. Apart from the uninteresting regime of extremely small macroion surface charge density (in which case the elongated shape of molecules is irrelevant, rendering our previous results applicable), charged rods adsorbed at the surface tend to be parallel to each other due to the strong lateral repulsion. In other words, there is the short range order of a one-dimensional Wigner crystal with lattice constant $A$ in the direction perpendicular to the rods (Fig. 8).

To make the signs consistent with the case of DNA, we assume that the polyelectrolyte charge is negative and equal to $-\eta$ per unit length, while the macroion surface is a plane with positive charge density $\sigma$. We assume also that there is a certain concentration of monovalent salt, $N_{1}$, in the solution, corresponding to the Debye screening radius (5). To begin with, let us assume that charge density of the rods, $-\eta$, is below the Onsager-Manning threshold, Eq. (7), and let us apply the Debye-Hückel approximation to describe the screening of the charged surface by the monovalent salt. We can then directly minimize the free energy of the one-dimensional crystal of negative rods on the positive surface written similarly to Eq. (17). Then the competition between the attraction of the rods to the surface and the repulsion of the neigh- boring rods results in the negative net surface charge density $-\sigma^{*}$ similar to Eq. (18) (Netz and Joanny, 1999a; Nguyen et al, 2000a):

$$
\frac{\sigma^{*}}{\sigma}=\frac{\eta / \sigma r_{s}}{\ln \left(\eta / \sigma r_{s}\right)}, r_{s} \ll A_{0} .
$$

Here the applicability condition involves $A_{0}=\eta / \sigma$, which is the distance between rods when they neutralize the plane; only at $r_{s} \ll A_{0}$ is the overcharged plane linearly screened by monovalent salt.

Speaking about DNA, we have already discussed in Sec. II that the DNA charge density, $-\eta$, is such that about three quarters of it is compensated by positive Onsager-Manning-condensed monovalent ions. In other words, the net charge of DNA in the bulk solution is $\eta^{*}=-\eta_{1}(7)$. It turns out that at $r_{s} \ll A_{0}$ the result (19) applies to DNA with the only correction of replacing $-\eta$ with $\eta^{*}=-\eta_{1} \equiv-k_{B} T \epsilon / e$ (Nguyen et al, 2000a).

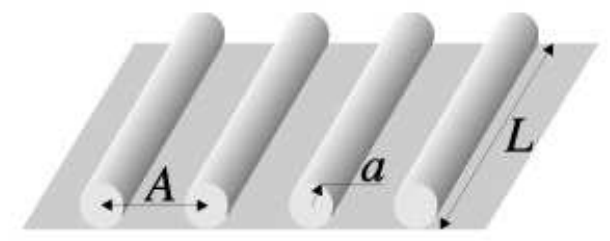

FIG. 8. Rod-like negative $Z$-ions, adsorbed on a positive uniformly charged plane.

Thus the inversion ratio grows with decreasing $r_{s}$ as in the case of spherical $Z$-ions. At small enough $r_{s}$ and $\sigma$, the inversion ratio can reach $200 \%$ before DNA molecules are released from the surface. It is larger than for spherical ions, because in this case, due to the large length of the DNA helix, the correlation energy remains large and the Wigner crystal-like short range order is preserved at smaller values of $\sigma r_{s}$. Nguyen et al (2000a) called this phenomenon "giant charge inversion."

Let us switch now to the opposite extreme of weak screening by a monovalent salt, $r_{s} \gg A_{0}$. In this case, screening of the overcharged plane by monovalent salt becomes strongly nonlinear, with the Gouy-Chapman screening length $\lambda^{*}=\epsilon k_{B} T /\left(2 \pi e \sigma^{*}\right)$ much smaller than $r_{s}$. Furthermore, some of the positive monovalent ions Onsager-Manning condensed on DNA are released from it upon adsorption, as the plane repels them (Park et al, 1999; Gelbart et al, 2000). As a result the absolute value of the net linear charge density of each adsorbed DNA, $\eta^{*}$, becomes larger than $\eta_{1}$. To determine $\sigma^{*}$ and $\eta^{*}$, Nguyen et al (2000a) considered two equilibrium conditions, dealing with chemical potentials of rods and small ions, respectively. As a result, they arrived at the following beautiful formulae valid at $r_{s} \gg A_{0}$ : 


$$
\begin{gathered}
\frac{\sigma^{*}}{\sigma}=\frac{\eta_{1}}{2 \pi a \sigma} \exp \left(-\sqrt{\ln \frac{r_{s}}{a} \ln \frac{A_{0}}{2 \pi a}}\right), \\
\eta^{*}=\eta_{1} \sqrt{\frac{\ln \left(r_{s} / a\right)}{\ln \left(A_{0} / 2 \pi a\right)}}, \quad r_{s} \gg A_{0} .
\end{gathered}
$$

At $r_{s} \simeq A_{0} / 2 \pi$ we get $\eta^{*} \simeq \eta_{1}, \lambda^{*} \simeq r_{s}$ and $\sigma^{*} / \sigma \simeq$ $\eta_{1} /\left(2 \pi r_{s} \sigma\right)$ so that Eq. (20) crosses over smoothly to the strong screening result of Eq. (19).

So far in this Section we assumed a rod-like polyelectrolyte. Let us now discuss how chain flexibility affects charge inversion. We argue that the results for rod-like $Z$-ions are remarkably robust. To begin with, consider a polyelectrolyte having several charged groups per each persistence length. We argue that our results remain valid as long as adsorption energy per one persistence length is large compared to $k_{B} T$. Indeed, under this condition even flexible polyelectrolyte chains lay flat on the surface, in which case they are ordered in Wigner crystallike SCL and, therefore, behave similarly to rods.

Dobrynin et al (2001) addressed the opposite extreme, namely, weakly charged polyelectrolytes, with so small a fraction of charged monomers, $f$, that a link between two neighboring charges is already a flexible polymer; in other words, the distance between charges is larger than the persistence length. It was discovered by de Gennes et al (1976) that a weakly charged polyelectrolyte chain in a bulk solution consists of electrostatic blobs. Inside each blob polymer is only marginally perturbed by Coulomb interactions, while chain of blobs is fully stretched, rod-like. Dobrynin et al (2001) have found that this blob structure remains valid for the adsorbed chains, which form SCL of effective rods of blobs. Speaking of charge inversion, this means that Eq. (19) remains valid for the weakly charged chains, provided $\eta$ is replaced with a linear charge density of the string of blobs $\eta^{*}=\left(f \epsilon e k_{B} T / l^{2}\right)^{1 / 3}$, where $l$ is the chain persistence length, and $r_{s}$ is larger than a blob size.

With increasing $\sigma$, adsorbed rods, either real or blob ones, start to touch each other leading to multilayer adsorption. It is only in this regime that the real and blob rods behave differently, as we discuss in Sec. VIII.

\section{POLYELECTROLYTES WRAPPING AROUND CHARGED PARTICLES}

As we have mentioned in the Introduction, one of the practically important situations is that of a long charged polymer forming complexes with oppositely charged particles. Motivated by the problem of nucleosome charge inversion, Mateescu et al (1999), Park et al (1999), Sens and Gurovich (1999), and Netz and Joanny (1999b) considered the complex of a positive sphere with charge $q$ and a negative polyelectrolyte, such as a DNA double helix, which has to make some $n_{t}>1$ turns around the sphere to neutralize it (Fig. 9). These authors predicted a substantial charge inversion: more of the polyelectrolyte is wound than is necessary to neutralize a sphere. Furthermore, Mateescu et al (1999) found that a tightly coiled polyelectrolyte conformation becomes unstable when the chain length exceeds a certain threshold, and then an almost straight tail abruptly stretches out (Fig. 9).

Nguyen and Shklovskii (2001a) emphasized the role of correlations in this case of charge inversion. Indeed, neighboring turns repel each other and form an almost equidistant solenoid, which locally resembles SCL. The tail of the polyelectrolyte repels the already adsorbed part of the polyelectrolyte and creates a correlation hole, which attracts the tail back to the surface (compare Fig. 6 ). As a result, the net charge of the sphere with wrapped polyelectrolyte, $q^{*}$, is negative. It is shown that at $r_{s} \rightarrow \infty$ the charge inversion ratio scales as $\left|q^{*}\right| / q \sim 1 / n_{t}$. On the other hand, at small enough $r_{s}$ it can exceed $100 \%$.

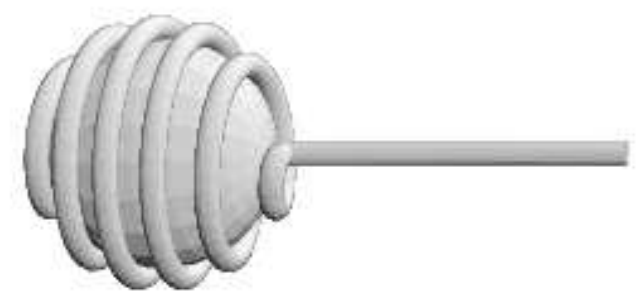

FIG. 9. A polyelectrolyte molecule winding around a spherical macroion. Due to the Coulomb repulsion, neighboring turns, which play the role of $Z$-ions, are strongly correlated.

Even more interesting is the system in which charged polymer is so long that it forms complexes with many oppositely charged particles (see Fig. 4). Examples include micelles (Wang et al, 1999), globular proteins (Kabanov et al, 1976; Xia and Dubin, 1994), colloids (Braun et al, 1998; Keren et al, 2001), or dendrimers (Kabanov et al, 2000; Evans et al, 2001), and, last but not least, histone octamers forming $10 \mathrm{~nm}$ chromatin fiber with DNA (Fig. 3). To be specific, we remain with the signs consistent with the DNA case and consider a long negative polymer chain in the solution of positive spheres. If the concentration of spheres is large, it is favorable to adsorb many spheres on the polyelectrolyte chain. As a result, each sphere is under-screened by polyelectrolyte and has positive net charge. Then, adsorbed spheres repel each other and the complex forms a periodic necklace (see Fig. 4). This necklace is, in fact, a one-dimensional Wigner crystal, or SCL, of spheres, which serve as $Z$ ions . Indeed, since the segment of the polyelectrolyte wound around one sphere interacts almost exclusively with this sphere, it plays the role of the Wigner-Seitz cell. Because of correlations, spheres bind to polyelec- 
trolyte in such a large number that the net charge of the polyelectrolyte molecule becomes positive (Nguyen and Shklovskii, 2001b). In this case, the charge inversion ratio scales as $Q^{*} / Q \sim n_{t}^{1 / 4}$ in the absence of monovalent salt, where $-Q$ and $Q^{*}$ are the bare and net charges of the polyelectrolyte molecule, respectively. This means that charge inversion may be larger than $100 \%$. As we discussed in Sec. V, charge inversion can be further enhanced by a monovalent salt, in which case $Q^{*} / Q \sim n_{t}$. We shall return to the complexes of a charged chain with spheres in Sec. IX.

\section{MULTILAYER ADSORPTION}

So far we have not considered the possibility that $Z$ ions fully cover the macroion surface. This may sometimes happen, particularly when a macroion is very strongly charged, or $Z$-ions are large. Suppose, for instance, that each $Z$-ion has a hard core of radius $a$. In this case, the effect of excluded volume of the hard cores of the $Z$-ions adds positive contributions to the surface pressure and chemical potential of a SCL (10) that are proportional to $k_{B} T$ and that diverge at the full coverage. Close to the full layer this term compensates and then over-compensates the negative Coulomb term $\mu_{W C}$, so that charge inversion disappears. Indeed, a full layer is incompressible (see Fig. 10b), and, unlike a partially filled layer (see Fig. 6 or Fig. 10a), it does not allow for the creation of an image-like correlation hole.

At even larger macroion charge, the second layer starts to form, launching a new wave of charge inversion. In the beginning, charge inversion is small because all the attraction of a new $Z$-ion approaching the surface is provided by a weak interaction with an inflated image in the emerging second layer, where once again $A \gg a$ (Fig. 10c). Continuing, Nguyen and Shklovskii (2001c) arrived at the prediction of an oscillating inverted charge $Q^{*}$ as a function of $Q$ (see Fig. 10), where charge inversion vanishes every time the top layer of $Z$-ions is full.

Another way to look at this phenomenon is to examine a metallic electrode screened by $Z$-ions, when the potential of the electrode is controlled instead of its charge. In this case, oscillations of charge inversion and compressibility lead to oscillations of capacitance of this electrode with the number of adsorbed layers of $Z$-ions. This is similar to oscillations of compressibility and magnetocapacitance in the quantum Hall effect, which are related to consecutive filling of Landau levels (Efros, 1988; Kravchenko et al, 1990; Eisenstein, 1992). In this sense, we deal with a classical analog of the quantum Hall effect.

To conclude this section, let us return to the adsorption of weakly charged polyelectrolytes that we discussed briefly in the Sec. VII. Dobrynin et al (2001) have shown that parallel chains of adsorbed blobs start touching each other above the same threshold surface charge density $\sigma_{e}=e f / l^{2}$, which corresponds to the onset of squashing blobs on the surface. As a result, these authors arrived at the conclusion that if weakly-charged chains are adsorbed on the surface with $\sigma>\sigma_{e}$, they form a polymer liquid. In this liquid, correlations and image formation are only due to the uppermost layer, with the thickness about that of an unperturbed blob. There are no oscillations of inverted charge; instead, charge inversion saturates at about one layer of blobs and remains unchanged afterwards.

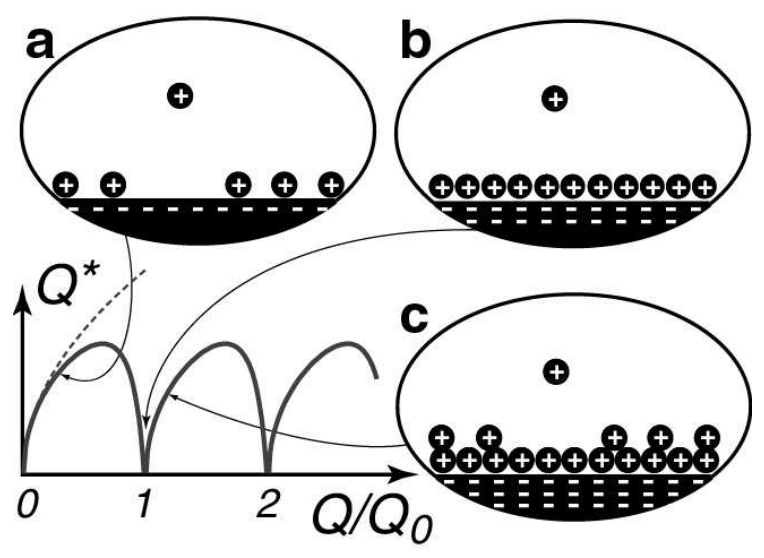

FIG. 10. Inverted charge $Q^{*}$ as a function of the absolute value $Q$ of the bare charge; $Q_{0}$ is the charge of one full layer of $Z$-ions. The dashed line corresponds to the case of $Z$-ions with vanishing radius, Eq. (14). (a) The first layer is not full, as in Fig. 6. An approaching new ion creates a correlation hole and is attracted to it. (b) The layer is full, there is no place for a correlation hole. (c) More than one layer is full. A correlation hole exists in the top layer only.

\section{CORRELATION-INDUCED ATTRACTION OF LIKE CHARGES}

The idea of a single screened macroion is a useful one in a theoretical context, but in practice it is rarely true that there is only one macroion. Typically, there is a certain concentration of them, so that interactions between them can be important. Let us start with the simplest question: consider two macroions, and suppose the concentration of $Z$-ions in solution is equal to $N_{0}$ (see Eq. (11)), such that each macroion forms a neutral complex with $Z$-ions. How do these two neutral complexes interact? It turns out that they attract each other at short distances and, therefore, tend to coagulate. In other words, two macroions of the same charge may attract each other because of the presence of $Z$-ions. In general, this attraction of like charges is as interesting a manifestation of correlations as charge inversion, even though our present Colloquium emphasizes the phenomenon of charge inversion. Nevertheless, we must discuss attraction at least 
briefly, in order to prepare the ground for the subsequent discussion of experiments (Sec. X).

Medium-induced attraction of like charges is nothing new in physics, with Cooper pairs of electrons being the most prominent example. In the context of molecular physics, the most popular explanation of attraction is in terms of salt bridges: a divalent ion, such as $\mathrm{Mg}^{2+}$, can form ionic bonds with two groups with charges of -1 each, in effect connecting them together. This idea is indeed adequate if we have, say, two macroion surfaces with regularly placed charges of -1 , and there are ions of charge +2 between them. However appealing, the bridge concept becomes increasingly fuzzy when $Z$ ions have charges of 3 or higher and when charges in the macroion are not positioned regularly.

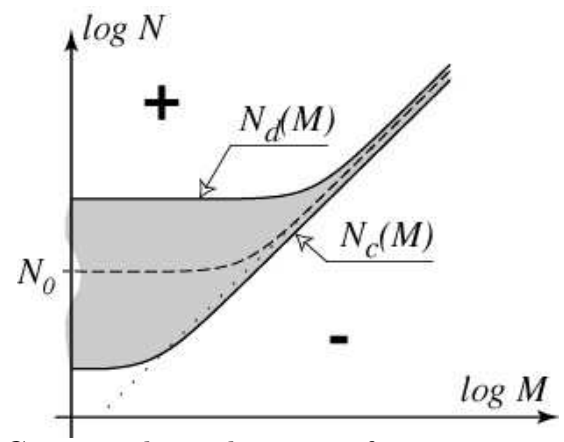

FIG. 11. Generic phase diagram of reentrant condensation and charge inversion in terms of macroion concentration $M$ and $Z$-ion concentration $N$ (Nguyen and Shklovskii, 2001d). Isoelectric composition is shown by the dotted line. The dashed "neutrality line" corresponds to neutral complexes in the dilute phase. The segregation region is shaded. Minus and plus indicate the signs of complexes of DNA with $Z$-ions.

Motivated by experimental observations of DNA condensation (see Sec. X), there was a significant effort by theorists to try to explain attractive forces by going beyond the bridge model. For simplicity, and following the majority of works, let us consider two planar macroion surfaces with some $Z$-ions between them. Of course, Poisson-Boltzmann theory predicts pure repulsion for such a system. However, attraction was observed in several computer experiments, including Guldbrand et al (1984), Kjellander and Marcelja (1985), GronbechJensen et al (1997), Linse and Lobaskin (1998), and Moreira and Netz (2000b). On the theory side, several groups attempted to go beyond the mean field approximation. An important observation is that due to dynamic fluctuations of counterions, there is an attractive component (similar to Van der Waals interactions), but at effectively high temperature, or small $\Gamma$ (see Eq. for the definition of (8)), the Poisson-Boltzmann repulsion still dominates and the force remains mainly repulsive (Oosawa, 1968; Lau and Pincus, 1998; Ha and Liu, 1998; Podgornik and Parsegian, 1998; Golestanian et al, 1999; Golestanian and
Kardar, 1999).

On the other hand, attraction of like charges dominates at effectively low temperatures, when $\Gamma \gg 1$, and the idea of spatial correlations between $Z$-ions, which is the central idea of this Colloquium, sheds light on the nature of this attraction. Indeed, for extremely large $\Gamma$, we deal with two Wigner crystals on the two opposing plates; they gain energy when approach each other by properly positioning themselves in the lateral direction. This was shown by Rouzina and Bloomfield (1996) (see also Gronbech-Jensen et al, 1997; Levin et al, 1999; Moreira and Netz, 2000b). Furthermore, Gronbech-Jensen et al (1997) and Shklovskii (1999a) pointed out that the long range order of a Wigner crystal is not important for this attractive force. As in the case of charge inversion, what is important is correlation and short range order. As we know, $Z$-ions form a SCL on the macroion surface as soon as $\Gamma$ becomes large. Imagine now bringing two planar surfaces, along with their respective SCL, very close to each other. What we have now is essentially two copies of Fig. 5 superimposed on top of one another, with $Z$-ions confined in between. Clearly, two SCL merge, lowering the energy per $Z$-ion from $\varepsilon(n)$ to $\varepsilon(2 n)<\varepsilon(n)$ (see Eq. (9)). Physically, every $Z$-ion in the merged SCL is sandwiched between two macroion surfaces, and its Wigner-Seitz cell can be approximated as a pair of discs, one on each surface (see again Fig 5). The charge of the cell must still be $-Z e$, but since there are two surfaces, the radius of the cell is reduced by the factor $1 / \sqrt{2}$, leading to the energy gain. In some sense, this theory returns us to the idea of bridges, albeit on a completely new level, with each $Z$-ion bridging between two sides of its Wigner-Seitz cell, which can include many surface charges.

These arguments hold, at least qualitatively, not only for plates, but also for macroions of other shapes, including DNA double helices. To be specific, consider two DNA double helices. When the concentration of $Z$-ions is equal to $N_{0}$, each DNA is neutralized by $Z$-ions, and the two neutral complexes attract each other at short distances. What happens if the concentration of $Z$-ions is higher or lower than $N_{0}$ ? In this case, correlationinduced attraction, which is short-ranged, competes with Coulomb repulsion, which is much longer-ranged. Note that the Coulomb repulsion force is present both at $N<N_{0}$, when the DNAs are partially screened by $Z$-ions and negative, and at $N>N_{0}$, when they are overcharged and positive.

What are the implications of this competition between attraction and repulsion? They are summarized in Fig. 11 which shows a phase diagram of the solution with number concentrations of macroions, $M$, and $Z$-ions, $N$ (along with the neutralizing amount of monovalent ions and salt.) The major feature of the phase diagram is the segregation region, which is the shaded area in the 
Fig. 11. As the figure indicates, the generic scenario is that of reentrant condensation. DNA molecules stay in solution and remain negative at $N<N_{c}(M)$, forming undercharged complexes with $Z$-ions. At some concentration of $Z$-ions, $N=N_{c}(M)$, repulsion loses to the correlation attraction and a condensed phase of DNA is formed, coexisting with a dilute phase. The condensed phase for DNA represents a (nematic) bundle of helices, it exists in the interval $N_{c}(M)<N<N_{d}(M)$. Finally, at $N=N_{d}(M)$, repulsion overcomes the correlation attraction, and the DNA molecules dissolve and form positive (overcharged) complexes with $Z$-ions.

Inside the coexistence region, there is a neutrality line, on which the equilibrium dilute phase consists of neutral complexes. At very small DNA concentrations, the neutrality condition corresponds to the concentration $N_{0}$ of $Z$-ions (Eq. 11). To see what happens at larger DNA concentration, consider increasing $M$, starting from the overcharged complexes, well above segregation on the phase diagram in Fig. 11. When $M$ grows, the solution runs out of $Z$-ions when it approaches the "isoelectric line" $-\eta L M+Z e N=0$, with $-\eta<0$ and $L$ being the DNA linear charge density and length, respectively. Near this line, the charge of complexes flips sign. Thus, the neutrality line crosses over from $N=N_{0}$ to the isoelectric line. The border lines $N_{c}(M)$ and $N_{d}(M)$ follow a similar pattern. Although not plotted in Fig. 11, at extremely small values of $M$ these two lines join together at a critical point, and for smaller $M$ only intramolecular condensation of DNA (the coil-globule transition) is possible if the DNA molecule is long enough.

We have considered the phase diagram in Fig. 11 for a solution of DNA chains with small $Z$-ions. As a matter of fact, the diagram is qualitatively quite general (Nguyen and Shklovskii, 2001d). For instance, it applies to a solution of DNA with large positively charged particles. In Sec. VII, we considered the case of a small DNA concentration, $M$, and a large concentration of spheres, $N$, which corresponds to the region above the coexistence region on the phase diagram in Fig. 11. We found that complexes have the form of necklaces, as shown in Fig. 4, and that they are overcharged, i.e., contain more spheres than necessary to neutralize DNA molecule. Large spheres are so strongly bound to DNA that the concentration $N_{0}$ for them is extremely small and any real experiment deals with the narrow upper-right part of the diagram. Suppose now that there are relatively few spheres in the solution, so we are below the neutrality line. In this situation, chains make an overcharging number of turns around each sphere. This is energetically favorable due to the repulsive correlations between subsequent turns on a sphere surface. The inverted net charge of each sphere is about as large as for the case of a single sphere discussed in Sec. VII. Furthermore, the inverted charge of spheres determines their distribution along the chain of polyelectrolyte. Negative spheres repel each other and, therefore, the complex once again has the periodic beads-on-a-string structure, Fig. 4, which resembles the $10 \mathrm{~nm}$ chromatin fiber. In the narrow vicinity of the neutrality line, even a small correlation attraction between touching spheres is sufficient to drive aggregation (or coil-globule collapse for long chains) of DNA with spheres.

\section{EXPERIMENTAL EVIDENCE OF CHARGE INVERSION}

How does the theory of correlated screening compare with experiment? For the purposes of this Colloquium, we restrict ourselves to a qualitative comparison only.

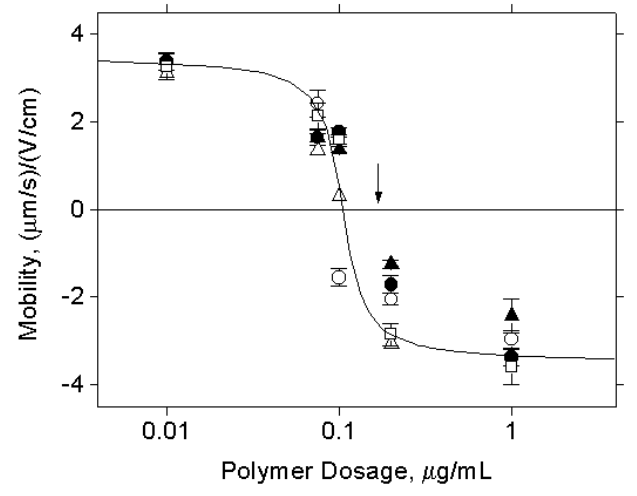

FIG. 12. Mobility of positive latex particles (macroions) in the presence of $0.005 \mathrm{M}$ (moles per liter) concentration of $\mathrm{NaCl}$ as a function of polymer mass concentration. Polymers (single-stranded DNA chains) play the role of $Z$-ions. Different symbols correspond to DNA of the following lengths (in monomers): $\Delta-8, \circ-10, \triangle-40, \bullet-80, \square-1400$. The line is drawn to guide the eye. The arrow indicates the isoelectric point, the polymer mass concentration of $0.17 \mu \mathrm{g} \mathrm{ml}^{-1}$ at which DNA charge neutralizes the latex particles.

First observations of charge inversion were reported a long time ago by De Jong (1949). He was able to measure electrophoresis of macroscopic aggregates in the phase segregation region of the phase diagram Fig 11, and observed the reverse of their mobility upon crossing the isoelectric line. More recently, Kabanov and his coworkers (Kabanov and Kabanov, 1995; Kabanov et al, 1996; Kabanov and Kabanov, 1998) examined mixtures of positive and negative polymers and directly observed the interpolyelectrolyte complexes in which a larger polymer of one sign (playing the role of a macroion) was seen to bind an overcharging amount of smaller polymers of the opposite sign (playing the role of $Z$-ions). The effect was directly seen due to the reversal of the electrophoretic mobility of complexes. Furthermore, Wang et al (1999) observed similar reversal for a mixture of poly- 
electrolytes (macroions) and micelles ( $Z$-ions). Gotting et al (1999) found the reversed mobility for the nanoparticles (macroions) and short single stranded DNA (elongated $Z$-ions). Walker and Grant (1996) demonstrated this phenomenon for $120 \mathrm{~nm}$ latex particles (macroions) with single stranded DNA ( $Z$-ions) ranging from 8 to 1400 nucleotides; their data are presented in Fig. 12. Kabanov et al (2000) and Evans et al (2001) observed the reversed electrophoretic mobility for DNA with the dendrimers as $Z$-ions.

An interesting observation, apparent from Fig. 12, is that the data for different DNA lengths collapse onto a single master curve upon re-scaling (in which mobility is plotted against mass concentration of DNA instead of number concentration, $N$ ). We note in passing that this observation can be rationalized by the following argument. Isoelectric point is obviously determined by the number of charged groups per unit volume, but this quantity is insensitive to the overall length of DNA and is simply proportional to the mass concentration.

All of the above mentioned experimental works rely on the reverse of electrophoretic mobility as an indication of charge inversion. Indeed, this is conceptually the most straightforward approach. It is valid because $Z$ ions are strongly bound to the macroion, with energies larger than $k_{B} T$, and move together with it. On the other hand, monovalent ions screening the net charge $Q^{*}$ are attracted to macroion with energy much smaller than $k_{B} T$ and move in electric field in the opposite direction. For these reasons, it is the net charge $Q^{*}$ that determines both magnitude and sign of the observed electrophoretic mobility. This remains correct also in the case when monovalent ions adsorb on $Z$-ions, effectively reducing them to $Z^{\prime}$-ions with $Z^{\prime}<Z$, as in Fig. 7. In all cases, the net charge includes all ions bound with energies in excess of $k_{B} T$. In a recent molecular dynamics simulation, Tanaka and Grosberg (2001b) have directly examined the mobility of charge inverted macroion complexes similar to the one shown in Fig. 7. They confirmed that adsorbed $Z$-ions drift together with the macroion in a weak electric field and that the sign of the net charge $Q^{*}$ determines the direction of electrophoresis.

It is also worth saying that the interpretation of electrophoretic experiments on charge inversion is not affected by the recent discoveries of Long et al (1996 and 1998). These authors noted that electrophoretic mobility, under some circumstances, may not be entirely determined by the charge. For instance, even an overall neutral object may move in an electric field provided that it has strong asymmetry of charge distribution. A simplest example consists of two balls of different radiuses, rigidly connected by a thin rod, and having opposite charges. The effect is due to the fact that external electric field acts not only on macroion itself, but also on surrounding co- and counterions, causing the latter to flow and to exert viscous friction drug forces on the macroion. Strong geometrical asymmetry of positive and negative charges leaves these drug forces unbalanced. In this colloquium, we discuss only macroions in which the bare charge is uniformly distributed on the surface. Then, the charge of $Z$-ions is practically uniform, too (apart from the small length scales of the order of $R$, the distance between neighboring adsorbed $Z$-ions). For such macroions, reversal of electrophoretic mobility does indeed indicate the inversion of charge.

For a more detailed comparison with experiments, we should remember that charge reversal is expected to be accompanied by coagulation, as discussed above in Sec. IX. Whether in equilibrium or not quite in equilibrium, these large complexes should scatter light strongly. There are many experiments reporting such observations.

Let us begin with DNA. It has been known for some time that at some critical concentration of $Z$-ions, $N_{c}$, DNA abruptly condenses into large bundles (Bloomfield, 1996). Recently it was discovered that at a much larger critical concentration, $N_{d}$, the bundles dissolve back (Saminathan et al, 1999; Pelta et al, 1996a; Pelta et al, 1996b; Raspaud et al, 1998; Raspaud et al, 1999). Specifically, for the spermine ions $(Z=4)$, it was found experimentally that $N_{c}=0.025 \mathrm{mM}$ and $N_{d}=150 \mathrm{mM}$. If one interprets these data in the framework of the theory of Nguyen et al (2000c), the experimental values of $N_{c}$ and $N_{d}$ imply that, for spermine, $N_{0}=3.2 \mathrm{mM}$ and the binding energy of two helices per one spermine ion is $u=0.3 k_{B} T$. The last value agrees with the one obtained by a different method (Rau and Parsegian, 1992).

Let us now discuss some other systems. Wang et al (1999) studied complex formation in mixture of micelles and oppositely-charged polyelectrolytes. In this experiment, the total charge of micelles was controlled by changing the concentration of the cationic lipid in the solution. In agreement with the above theory, measurements of dynamic light scattering and turbidity (coefficient of light scattering) show that complexes condense in bundles and the solution coacervates in the vicinity of the point where mobility crosses over between two almost constant values, positive and negative.

For the complexes of latex particles with DNA of various lengths, examined by Walker and Grant (1996), equilibrium conditions were not found, but a significant rate of aggregation of latex particles was observed in the same narrow range of DNA concentrations where the mobility flips the sign.

There is a large body of interesting experimental (Rädler et al, 1997; Koltover et al, 1999) and theoretical (Harries et al, 1998; Bruinsma, 1998) work on phase diagrams and overcharging of lamellar cationic lipidDNA self-assembled complexes. These solutions always seem to have aggregates due to hydrophobic attraction of lipids. A phase diagram of this kind has been sketched 
by Rädler (2000).

Another large group of works with nice (and technologically useful) examples of overcharging deals with the sequential adsorption of multiple layers of polyelectrolytes of alternating sign (see Decher (1997) and references therein). Say, a positive surface is first treated with negative polymers, gets overcharged and becomes negative, then it is treated with positive polymers, gets overcharged etc. This procedure works reliably with up to many tens of layers. Theoretical interpretation of this technique was discussed by Castelnovo and Joanny (2000). It falls beyond the framework of the present Colloquium as it involves certain kinetic considerations while we deal in this Colloquium only with equilibrium phenomena.

\section{CORRELATIONS "IN A SHEEP'S SKIN"}

We mentioned correlations so many times in this Colloquium, that the reader may want to ask: Are there alternative, correlation-independent, electrostatic mechanisms leading to charge inversion? Our answer is no, and we argue that the correlation-based mechanism is the universal one. This fact notwithstanding, we should say that correlations may show up in a number of ways, sometimes masked like a wolf in a sheep's skin.

To understand better the role of correlations, let us first consider the case of no correlations. Namely, suppose, we have a set of randomly positioned point-like charges, equal numbers of $+e$ and $-e$. It is easy to establish that the averaged interaction energy in such system is exactly zero, where average is taken over independent random positions of all charges. Similarly, averaged electric field in the system is also zero. Both these statements remain correct also for the one component plasma, in which point-like charges of one sign are randomly positioned on the smeared uniform background of the opposite sign.

The essence of screening is that charges in plasma are not positioned randomly. Correlations happen because ions reconfigure themselves non-randomly to gain some energy. This so-called correlation energy is well known in plasma physics (Landau and Lifshitz, 1977). It is negative, meaning that correlated configuration is lower in energy, or more thermodynamically favorable, than random configuration.

These simple facts allow us to understand the underlying role of correlations in one of the theories suggested in the literature to explain charge inversion. This theory, put forward by Park et al (1999), views monovalent counterion release from $Z$-ions as the driving force behind charge inversion. We argue that while counterion release accompanies, it is itself driven by correlations. As we did already more than once, let us imagine that DNA molecules, along with their Onsager-Manning-condensed small ions, are being adsorbed on the macroion one at a time (possibly releasing some of their counterions). Let us further consider the moment when the neutralization condition has just been achieved. Pretend now that DNA rods ( $Z$-ions) are distributed randomly on the surface, uncorrelated in both positions and orientations. In this case, next arriving DNA molecule feels no average field, so that it has no reason to release its counterions. The situation is completely different if DNA molecules are correlated on the surface (see Figs. 2 or 8), where locally each molecule is surrounded by a correlation hole - the positive stripe of the background charge (the Wigner-Seitz cell). The corresponding field, or the positive potential of the Wigner-Seitz cell, causes the release of counterions from DNA not only at the neutrality point, but even if the surface overall is overcharged (see the solution of this problem given by Eqs. (20), (21)). These qualitative arguments can be formulated also more quantitatively (Nguyen et al, 2001). Thus, correlation hole, or adjustment of DNA molecules to each other, or image charge, or correlations (all synonyms!) is the driving force for both counterion release and charge inversion; under a sheep's skin of counterions release, there is a wolf's face of correlations.

Let us now discuss another approach which we call metallization. It was pioneered by Mateescu et al (1999), who considered complexation of a polyelectrolyte with a sphere, and by Joanny (1999), who examined adsorption of flexible polymers on a charged plane. Metallization theory considers adsorbed $Z$-ions as a continuous medium similar to a metal, while still treating the bulk solution as consisting of discrete charges.

We argue that metallization theory is in fact an overestimate of correlation effects. Indeed, as we illustrated with Fig. 6, SCL behaves as a metal on the length scale above $R$, the distance between neighboring adsorbed $Z$ ions. It behaves as a metal in the sense that it responds to the approaching new ion by forming an image. Clearly, the smeared continuum is also a metal in this sense, and even a better metal - it is a metal on all length scales. Another way to view that same physics is to note that correlation suppresses electric field of every $Z$-ion beyond certain distance of order $R$, while for the smeared continuum the field is suppressed everywhere and, therefore, the effect is overestimated.

The latter view suggests also another fruitful interpretation of metallization approach, which is in terms of the self-energy of $Z$-ions. Here, we resort to the terminology in which self-energy of an ion is identified with the energy of the electric field outside certain cutoff length, such as an ion size. (We are not interested in the electric field on the smaller length scales: although energy of this field is infinite, it does not change upon any processes considered here, such as adsorption of $Z$-ions.) Using 
this language, the metallization theory becomes physically transparent. Indeed, in this theory adsorption of $Z$-ions is energetically favorable because they do have self-energy while in the bulk solution and loose it completely upon adsorption. Once again, real screening, or correlations between $Z$-ions, correspond to suppression of a part of self-energy, corresponding to the energy of the electric field beyond the distance of order $R$. Energy of the field between the ion size and $R$ is the amount of overestimation by the metallization theory. By contrast, the Poisson-Boltzmann approximation fails to describe charge inversion precisely because it smears $Z$-ions everywhere and neglects their self-energies.

Representation of correlations in terms of self-energy allows us to address one more practically important problem, namely, the discreteness of charges (Nguyen and Shklovskii, 2001e). Indeed, instead of uniformly charged surface, like Fig. 1, it would be closer to reality (and to a chemist's heart) to think of a macroion as having some charged groups with unitary charge each. For instance, negative $(-e)$ charges of DNA are positioned on an external spiral rim of the double helix, at the distance $A=0.67 \mathrm{~nm}$ from each other along the rim.
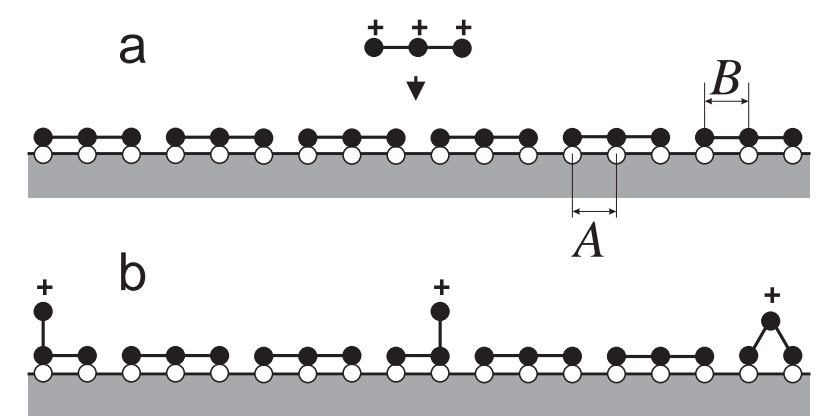

FIG. 13. Schematic representation of charge fractionalization. a) One strand of negative charges of DNA (empty balls) is completely neutralized by positive $Z$-ions with $Z=3$, their charged groups are shown by black balls. A new $Z$-ion is approaching. b) The new $Z$-ion is "digested." Its charge is split in $+e$ charges of $Z$ defects, tails and archs.

To be specific, let us consider a macroion which is a regular lattice of charges $-e$ ("unfolded DNA strand") and $Z$-ion having also a linear array of charges $+e$ (a short polyelectrolyte). Importantly, $Z$-ions always have some degree of flexibility; for instance, in the case $Z=3$, as shown in Fig. 13, we can imagine that they freely bend in the middle. For simplicity we assume that the distance between charges in the $Z$-ion, $B$, matches exactly that in the macroion, $A: A=B$. Finally, we assume that the $Z$-ion charges and the macroion charges can approach each other to the minimal distance much smaller than $A$. Then $Z$-ions can attach to the macroion locally compensating each charge and, therefore, achieving complete neutralization, as we show in Fig. 13a. The neutralization is so perfect that it is difficult to imagine how another $Z$-ion can be attached. Fig. 13 explains why this happens. Similarly to Fig. 6 , suppose that the macroion is already neutralized by $Z$-ions and a new $Z$ ion comes. Then it turns out energetically favorable to disturb order among the neutralizing $Z$-ions by $Z$ defects in $Z$ independent places, thus opening a room for a new $Z$-ion. In each defect, one charge of $Z$-ion is detached from the corresponding macroion charge, forming positive tail or arch above the surface and leaving negative vacancy on the macroion. Then, shifting $Z$-ions along the macroion, $Z$ vacancies can join together and form a large vacancy capable to accommodate an entire new $Z$-ion. A net result is that $Z$ disconnected charges $+e$ appear on top of completely neutralized macroion (Fig. 13b), or, in other words, the charge of $Z$-ion is fractionalized. To avoid misunderstanding, we emphasize that none of the chemical bonds is really cut.

Fractionalization effectively eliminates self-energy of the free $Z$-ion. Indeed, self-energy of the $Z$-ion is simply the energy of repulsion between $Z$ positive constituent charged groups in the extended conformation which the $Z$-ion assumes in the solution. In the fractionalized state, charges are far apart and practically do not interact, so that self-energy is gained. These results are easily generalized for the case $B<A$, when $Z$-ions have larger linear charge density than macroion. For instance, if $B=A / 2$, then $Z$-ions repelling each other form a "Wigner crystal" on top of the lattice of macroion charges where they alternate with vacant places (similarly to Figs. 1, 5, 6, and 8).

Is fractionalization a correlation-independent mechanism of charge inversion? Of course, not: this phenomenon is solely due to the correlated distribution of $Z$-ions, which avoid each other at the macroion. Fractionalization is yet another mask under which correlations may show up.

\section{CHARGE INVERSION IN A BROADER PHYSICS CONTEXT}

In conclusion, we would like to show that charge inversion studied in this Colloquium has many physical analogies. There are other "charge inverted" systems in physics. Let us start from the hydrogen atom. It is known that it can bind a second electron, forming the negative ion $\mathrm{H}^{-}$with an ionization energy of approximately 0.05Ry (Massey, 1938). We can consider this effect as the inversion of proton charge. Attraction of the second electron to the neutral atom is due to the Coulomb correlation between electrons: the first electron avoids the second one, spending more time on the opposite side of the proton. In other terms one can say that binding is related to polarization of the neutral core.

Negative ions - nuclei overcharged by electrons - exist also for larger atoms. Mean field Thomas-Fermi or 
Hartree theories fail to explain negative ions (Landau and Lifshitz, 1980). One must include exchange and Coulomb correlation holes to arrive at a satisfactory theory explaining bound state and the nonzero ionization energy of a negative ion (Massey, 1938). The Thomas-Fermi theory of an atom is an analog of the Poisson-Boltzmann theory of electrolytes. It is not surprising that both fail to explain charge inverted states.

Similar considerations apply also for a macroscopic metallic particle. Electrons in such a particle have a negative (compare to vacuum) chemical potential or, in other words, positive work function. The work function is known to vanish in the Thomas-Fermi or Hartree approximations (Lang, 1973).

The energy $\left|\mu_{W C}\right|$ plays the same role for $Z$-ions on the insulating macroion surface as the ionization energy of a negative ion or the work function of metallic particle for electrons. Similarly to electrons, charge inversion of a charged insulating macroion by $Z$-ions can not be obtained in the mean field Poisson-Boltzmann approximation. Only correlations of $Z$-ions on the surface of the macroion can lead to charge inversion.

Let us now return to the Onsager-Manning condensation (Manning, 1969; Sec. II). Kosterlitz and Thouless (1972) discovered a similar threshold phenomenon for generation of free vortexes in two dimensional superfluids or superconductors. They noticed that due to the logarithmic form of attractive interaction, two vortexes of opposite sign decouple only above some critical temperature, $T_{K T}$. Later, Kosterlitz-Thouless theory was applied to unbinding of dislocations and disclinations in the theory of defect-induced melting of two-dimensional crystals (Nelson and Halperin, 1979; Young, 1979).

In the Kosterlitz-Thouless theory one can identify ana$\log$ of the short-range correlation contribution to the chemical potential of $Z$-ions (which we called $\left|\mu_{W C}\right|$ ). This is the energy of creation of the two vortex cores. Similarly to $\left|\mu_{W C}\right|$, this energy provides additional binding of vortexes and strongly reduces the concentration of free vortexes at $T>T_{K T}$ (Minnhagen, 1987). In contrast to the Kosterlitz-Thouless theory, the short range contribution $\left|\mu_{W C}\right|$ was only recently introduced (Perel and Shklovskii, 1999).

Another physical analogy already mentioned in Sec. VIII is the integer quantum Hall effect. We should add that charge fractionalization, as illustrated by Fig. 13, is an analog of the fractional quantum Hall effect (Prange and Girvin, 1990). Finally, Fig. 13 bears also analogy with electron charge fractionalization in the polyacetilene (Brazovskii and Kirova, 1991).

\section{CONCLUSIONS AND OUTLOOK}

To conclude, we have discussed the physical picture of screening for the case of strongly interacting ions.
This case appears to have been overlooked for many decades, since Debye. Its theoretical study, motivated by the experiments mainly on gene delivery and chromatin structure, revealed new interesting physical insights. Specifically, correlations between screening ions lead to such counterintuitive phenomena as charge inversion, reverse electrophoretic mobility, attraction of like charged molecules or colloids, etc. The physical theory of these phenomena is aesthetically attractive as it presents many parallels with other areas of physics, ranging from quantum Hall effect to atomic physics and metals. The potential applications are many, in both chemical and biological realms. We can mention here all sorts of manipulations with DNA, both for biological purposes and for using DNA as an assembly tool for non-biological nanotechnology. They all require understanding of electrostatic properties of DNA chains. Many diseases have to do with mis-assemblies of charged proteins, such as actin; we need to understand better the assembly of such objects. Food, cosmetic, paper, and waste water treatment industries, are all about charged colloids, and the list of applications is easy to continue. In brief, this theory is one of the busy junctions where physics meets chemistry and biology.

\section{ACKNOWLEDGMENTS}

We enjoyed collaboration with V. I. Perel, I. Rouzina, and M. Tanaka. We are grateful to V. Bloomfield, E. Braun, R. Bruinsma, P. Chaikin, A. Dobrynin, P. Dubin, M. Dyakonov, W. Gelbart, S. Girvin, W. Halley, C. Holm, J.-F. Joanny, A. Kabanov, V. Kabanov, A. Khokhlov, R. Kjellander, K. Kremer, A. Koulakov, V. Lobaskin, F. Livolant, D. Long, G. Manning, R. Netz, P. Pincus, R. Podgornik, E. Raspaud, M. Rubinstein, J.-L. Sikorav, U. Sivan, M. Voloshin, and J. Widom for useful discussions. We thank Zhifeng Shao for the permission to use Figs. 2 and 3 and S. Grant for the permission to use Fig. 12. T. T. N. and B. I. S. are supported by NSF DMR-9985785.

\section{REFERENCES}

Andelman, D., and J.-F. Joanny, 2000, cond-mat/0011072.

Aberts, B., D.Bray, J.Lewis, M.Raff, K.Roberts, J.D.Watson, 1994, Molecular Biology of the Cell, Galland Publishing, New York.

Bloomfield, V. A., 1996, Current Opinion in Structural Biol., 6, 334; 1998, Biopolymers, 44, 269; Ch. 7 at http://biosci.umn.edu/biophys/BTOL/supramol.html

Bjerrum, N. J., 2000, Zs. f. Phys. Chem., 119, 145.

Braun, E., Y. Eichen, U. Sivan, G. Ben-Yoseph, 1998, Nature, 391, n. 6669, 775. 
Brazovskii, S., N. Kirova, 1991, JETP Lett. 33, 4.

Bruinsma, R., 1998, Europ. Phys. J. B4, 75.

Castelnovo, M., and J.-F. Joanny, 2000, Langmuir 16, 7524.

Chapman, D., 1913, Philos. Mag. Sixth Ser. 25, 475.

Chodanowski, P., and S. Stoll, 2001, Macromolecules 34, 2320.

Debye, P., and E. Hückel, 1923, Phys. Zeitsch. 24, 185.

Decher, G., 1997, Science 277, 1232.

Dobrynin, A. V., A. Deshkovski, and M. Rubinstein, 2001, Macromolecules 34, 3421.

Efros, A. L., 1988, Solid State Comm. 65, 1281.

Eisenstein, J. P., L. N. Pfeifer, and K. W. West, 1992, Phys. Rev. Lett. 68, 674.

Ennis, J., S. Marcelja, and R. Kjellander, 1996, Electrochim. Acta 41, 2115.

Evans, H. M., A. Ahmad, T. Pfohl, A. Martin, C. R. Safinya, 2001, Bull. APS 46, 391.

Fang, Y., and J. Yang, 1997, J. Phys. Chem. B101, 441.

Felgner, P.L., 1997, Scientific American 276, 102.

Frank-Kamenetskii, M. D., V. V. Anshelevich, and A. V. Lukashin, 1987, Soviet Physics Uspekhi 30, 317.

Gelbart, W., R. Bruinsma, P. Pincus, and A. Parsegian, 2000, Physics Today 53, 38.

De Gennes, P.-G., P. Pincus, R. M. Velasco, F. Brochard, 1976, J. de Physique (Paris), 37, 1461.

Golestanian, R., M. Kardar, and T. B. Liverpool, 1999, Phys. Rev. Lett. 82, 4456.

Golestanian, R., and M. Kardar, 1999, Rev. Mod. Phys. 71, 1233.

Götting, N., H. Fritz, M. Maier, J. von Stamm, T. Schoofs, and E. Bayer, 1999, Colloid Polym. Sci. 277, 145.

Gouy, G., 1910, J. Phys. Radium 9, 457.

Gronbech-Jensen, N., R. J. Mashl, R. F. Bruinsma, and W. M. Gelbart, 1997, Phys. Rev. Lett. 78, 2477.

Guldbrand, L. G., Bo Jonsson, H. Innerstrom, and P. Linse, 1984, J. Chem. Phys. 80, 2221.

Ha, B.-Y., and A. J. Liu, 1998, Phys. Rev. Lett. 81, 1011; 2000, "Physical Chemistry of Polyelectrolytes," ed. T. Radeva, Marcel Dekker (New York); cond-mat/0003162.

Harries, D., S. May, W. M. Gelbart, A. Ben-Shaul, 1998, Biophys. J. 75, 159.

Joanny, J.-F., 1999, Europ. J. Phys. B 9, 117.

M. G. Bungenberg de Jong, 1949, in "Colloid Science", edited by H. R. Kruyt (Elsevier, New York)

Kabanov, V. A., V. P. Evdakov, M. I. Mustafaev, and A. D. Antipina, 1976, Molekulyarnaya Biologia 1152.

Kabanov, A. V., and V. A. Kabanov, 1995, Bioconjug. Chem. $6,7$.

Kabanov, V. A., A. A. Yaroslavov, S. A. Sukhisvili, 1996, J. of Control Release 39, 173.

Kabanov, A. V., and V. A. Kabanov, 1998, Advanced Drug Delivery Reviews 30, 49.

Kabanov, V. A., V. G. Sergeyev, O. A. Pyshkina, A. A. Zinchenko, A. B. Zezin, J. G. H. Joosten, J. Brackman, and K. Yoshikawa, 2000, Macromolecules 33, 9587.

Katzir-Katchalsky, A., 1971, Biophysics and Other Topics: Selected Papers, Academic Press, Orlando.

Keren, K., Y. Soen, G. Ben Yoseph, R. Yechieli, E. Braun, U. Sivan, and Y. Talmon, 2001, preprint.

Kjellander, R., and S. Marcelja, 1985, Chem. Phys. Lett. 114,
$124(\mathrm{E})$.

Koltover, I., T. Salditt, and C. R. Safinya, 1999, Biophys. J. 77, 915 .

Kosterlitz, J. M., and D. J. Thouless, 1972, J. Phys C 5, L124; 1973, J. Phys C 6, 1181.

Kravchenko, S. V., D. A. Rinberg, S. G. Semenchinsky, and V. Pudalov, 1990, Phys. Rev. B 42, 3741.

Landau, L. D., and E. M. Lifshitz, 1977, Statistical Physics, Part 1 (Pergamon Press, Oxford), Ch. VII.

Landau, L. D., and E. M. Lifshitz, 1980, Quantum Mechanics (nonrelativistic theory) (Pergamon Press, Oxford), Ch. X.

Lang, N. D., 1973, Solid state physics, Edited by H. Ehrenreich, F. Seitz, and D. Turnbull, (Academic Press, New York), Vol. 28.

Lau, A. W. C., P. A. Pincus, 1998, Phys. Rev. Lett 81, 1338.

Levin, Y., J. J. Arenzon, and J. F. Stilck, 1999, Phys. Rev. Lett. 83, 2680.

Linse, P., and V. Lobaskin, 1998, Phys. Rev. Lett., 83, 4208.

Long, D., J.-L. Viovy, and A. Ajdari, 1996, Phys. Rev. Lett. 76, 3858 .

Long, D., A. V. Dobrynin, M. Rubinstein, and A. Ajdari, 1998, J. Chem. Phys. 108, 1234.

Luger, K., A. Mader, R. Richmond, D. Sargent, T. Richmond, 1997, Nature, 389, 251. For pictures visit, e.g. http://info.bio.cmu.edu/Courses/03438/Nsome/1AOI.htm

Manning, G.S., 1969, J. Chem. Phys. 51, 924.

Massey, H.S.W., 1938, Negative Ions, Cambridge University Press.

Mateescu, E. M., C. Jepperseni, and P. Pincus, 1999, Europhys. Lett. 46, 454.

Messina, R., C. Holm, and K. Kremer, 2000, Phys. Rev. Lett. 85, 872; 2000, Europhys. Lett. 51, 461.

Minnhagen, P., 1987, Rev. Mod. Phys 59, 1001.

Moreira, A. G., R. R. Netz, 2000a, Europhys. Lett. 52, 705.

Moreira, A. G., R. R. Netz, 2000b, cond-mat/0009377.

Moreira, A. G., R. R. Netz, 2001 Europhys. Lett. (in press).

Mou, J., D. M. Czajkowsky, Y. Zhang, and Z. Shao, 1995, FEBS Letters 371, 279.

Nelson, D. R., and B. I. Halperin, 1979, Phys. Rev. B 19, 2457.

Netz, R. R., and J.-F. Joanny, 1999a, Macromolecules, 32, 9013.

Netz, R. R., J.-F. Joanny, 1999b, Macromolecules, 32, 9026.

Nguyen, T. T., A. Yu. Grosberg, and B. I. Shklovskii, 2000a, Phys. Rev. Lett. 85, 1568.

Nguyen, T. T., A. Yu. Grosberg, and B. I. Shklovskii, 2000b, J. Chem. Phys. 113, 1110.

Nguyen, T. T., I. Rouzina, B. I. Shklovskii, 2000, J. Chem. Phys. 112, 2562.

Nguyen, T. T., A. Yu. Grosberg, and B. I. Shklovskii, 2001, in Electrostatic Effects in Soft Matter and Biophysics, Proceedings of the NATO Advanced Study Institute, Les Houches, France, 1-13 October 2000, C. Holm, P. Kekicheff, R. Podgornik eds, NATO SCIENCE SERIES: II: Mathematics, Physics and Chemistry, Volume 46, Kluwer Academic Publishers, Dordrecht.

Nguyen, T. T., and B. I. Shklovskii, 2001a, Physica A 293, 324.

Nguyen, T. T., and B. I. Shklovskii, 2001b, J. Chem. Phys. 114, 5905 . 
Nguyen, T. T., and B. I. Shklovskii, 2001c, Phys. Rev. E 64, 041407.

Nguyen, T. T., and B. I. Shklovskii, 2001d, J. Chem. Phys. 115, 7298.

Nguyen, T. T., and B. I. Shklovskii, 2001e, Phys. Rev. ; condmat/0109002

Onsager, L., 1967, private communication to G. Manning.

Oosawa, F., 1968, Biopolymers 6, 134.

Oosawa, F., 1971, Polyelectrolytes, Marcel Dekker, NY.

Pan, J., D. Thirumalai, S. Woodson, 1999, Proc. Natl. Acad. Sci., 96, 6149.

Park, S. Y., R. F. Bruinsma, and W. M. Gelbart, 1999, Europhys. Lett. 46, 493.

Pelta, J., D. Durand, J. Doucet, and F. Livolant, 1996, Biophys. J. 71, 48.

Pelta, J., F. Livolant, and J.-L. Sikorav, 1996, J. Biol. Chem. 271, 5656.

Perel, V. I., and B. I. Shklovskii, 1999, Physica A 274, 446.

Podgornik, R., and V. A. Parsegian, 1998, Phys. Rev. Lett. 80, 1560.

Potemkin, I. I., K. B. Zeldovich, A. R. Khokhlov, 2000, Polymer Science, Ser. C, 42, 154.

Prange, R. E., and S. M. Girvin, eds., 1990, The Quantum Hall Effect (Springer, Heidelberg), 2nd edition.

Rädler, J. O., I. Koltover, T. Saddit, and C. R. Safinya, 1997, Science, 275, 810.

Rädler, J. O., 2000, in Electrostatics effects in Biophysics and soft matter, C. Holm, P. Kekicheff, R. Podgornik eds, Kluver.

Raspaud, E., M. Olvera de la Cruz, J.-L. Sikorav, and F. Livolant, 1998, Biophys. J. 74, 381.

Raspaud, E., I. Chaperon, A. Leforestier, and F. Livolant, 1999, Byophys. J. 77, 1547.

Rau, D. C., and A. V. Parsegian, 1992, Biophys. J. 61, 246.

Rouzina, I., and V. A. Bloomfield, 1996, J. Phys. Chem. 100, 9977.

Saminathan, M., T. Antony, A. Shirahata, L. Sigal, T. Thomas, and T. J. Thomas, 1999, Biochemistry 38, 3821.

Sens, P., and E. Gurovitch, 1999, Phys. Rev. Lett. 82, 339.

Shao, Z., 1999, News in Physiological Sciences, 14, 142.

Shklovskii, B. I., 1999a, Phys. Rev. Lett. 82, 3268.

Shklovskii, B. I., 1999b, Phys. Rev. E 60, 5802.

Tanaka, M., and A. Yu. Grosberg, 2001, J. Chem. Phys. 115, 567.

Tanaka, M., A. Yu. Grosberg, 2001, cond-mat/0106561.

Tang, M. X., C. T. Rederman, F. C. Szoka JR, 1996, Bioconjugate Chem. 7, 703.

Terao, T., and T. Nakayama, 2001, Phys. Rev. E 63, 041401.

Totsuji, H., 1978, Phys. Rev. A 17, 399.

Walker, H. W., and S. B. Grant, 1996, Colloids and Surfaces A, 119, 229.

Wallin, T., P. Linse, 1996, J. Phys. Chem. 100, 17873; 1997 ibid 101, 5506.

Wang, Y., K. Kimura, Q. Huang, P. L. Dubin, and W. Jaeger, 1999, Macromolecules 32, 7128.

Woodson, S., 2000, Nature Structural Biology, 7, 349.

Xia, J., and P. L. Dubin, 1994, Macromolecular complexes in Chemistry and Biology P. L. Dubin et al. eds. (SpringerVerlag, Berlin).

Young, A., 1979, Phys. Rev. B 19, 1855.
Zimm, B., and M. Le Bret, 1983, J. Biomol. Struct. and Dyn. 1, 461 . 